\title{
Is There any Impact of Local Endometrial Injury on Implantation and Clinical Pregnancy Rates in the First in Vitro Fertilization Cycles
}

\section{(D) Zeliha FIRAT CUYLANa, (D) Berfu DEMIR ${ }^{\mathrm{a}}$, (1) Berna DILBAZa (D) Serdar DíLBAZa \\ ${ }^{a} \mathrm{Clinic}$ of Obstetrics and Gynecology, Etlik Zübeyde Hanım Women's Health Training and Research Hospital, Ankara, TURKEY}

Received: 26 Aug 2019

Received in revised form: 15 Nov 2019 Accepted: 22 Nov 2019

Available online: 29 Nov 2019

Correspondence:

Zeliha FIRAT CUYLAN

University of Health Sciences Zekai Tahir Burak Women's Health

Training and Research Hospital,

Clinic of Gynecologic Oncology,

Ankara, TURKEY

zelihafiratcuylan@gmail.com

\begin{abstract}
Objective: To evaluate the impact of local endometrial injury (LEI), applied during the first in vitro fertilization (IVF)-embryo transfer (ET) cycle using office hysteroscopy, on implantation and pregnancy rates. Material and Methods: A total of 372 women under the age of 40 , who had their first IVF-ET treatment cycle, with no ovulatory or intracavitary pathology were included in this study. LEI was created in the fundus, anterior and posterior endometrial walls during office hysteroscopy, which was performed prior to the IVF-ET cycle. Long luteal gonadotropin releasing hormone agonist protocol was used in all cycles and 273 patients underwent embryo transfer. Implantation and pregnancy rates were compared to the control group. Results: Age, body mass index, baseline follicle stimulating hormone, luteinizing hormone, and estradiol levels, antral follicle counts, infertility etiologies, total oocyte counts, intra-cytoplasmic sperm injection percentages were similar, both in the study and the control groups. Clinical pregnancy rates $(28.7 \%$ vs $26.1 \%$, $\mathrm{p}>0.05)$, total pregnancy rates $(43.1 \%$ vs $38 \%, \mathrm{p}>0.05)$ and implantation rates $(19 \%$ vs $12.9 \%$, $\mathrm{p}>0.05)$ of the LEI injury group were higher than those of the control group, however the difference was not statistically significant. There was no statistically significant difference between the miscarriage rates. Conclusion: Even if implantation and pregnancy rates improved slightly in LEI group, the study did not reveal a significant improvement for the implantation and pregnancy rates after the LEI, which was created during hysteroscopy before the first IVF-RT treatment cycle.
\end{abstract}

Keywords: Embryo transfer; implantation; in vitro fertilization; local endometrial injury; office hysteroscopy

I mplantation is one of the most crucial steps for a successful in-vitro fertilization (IVF)-embryo transfer (ET). Implantation success depends on the quality of the embryo and endometrial receptivity. Even with a good quality embryo transfer, the rates of implantation may vary between 25$30 \%$ per transferred embryo. ${ }^{1,2}$ Implantation occurs during the "implantation window", which is the period between the $19^{\text {th }}$ and $23^{\text {rd }}$ days of the menstrual cycle, when endometrial receptivity is at its peak, and includes a three-stage process of apposition, adhesion and invasion of the blastocyst to the endometrium.

Recently, local endometrial injury (LEI) has been proposed as one of the methods that aim to increase the implantation rates in IVF-ET cycles. Initially, Barash et al. showed an increase in implantation and pregnancy rates in 134 women who had repeated endometrial biopsies as a method of LEI before IVF-ET cycles. ${ }^{3}$ Likewise, other studies have also demonstrated an increase in pregnancy rates after LEI in women with recurrent IVF failure. ${ }^{4,5}$ The first study investigating the effect of LEI on implantation and 
pregnancy rates in patients at the first IVF-ET cycle was presented by Karimzade et al. However, the therapeutic impact of LEI still remains unclear due to the heterogeneity of the patient population, timing of this intervention and techniques for inducing endometrial injury. ${ }^{6-8}$

The purpose of the present study was to demonstrate the effect of LEI, applied before the first IVF-ET cycle, using office hysteroscopy, on implantation and pregnancy rates.

\section{MATERIAL AND METHODS}

This retrospective case-control study was designed after the approval from the Local Institutional Review Board. Electronic medical records of the patients who were treated in the IVF Clinic of Etlik Zübeyde Hanım Women's Health Training and Research Hospital, Ankara, Turkey between June 2007 and November 2012 were analyzed retrospectively. All patients provided informed consents for the procedure and allowed use of their medical information for research purposes at the time of admission. In this study, the patients with the first IVF-ET cycle, under the age of 40 years, who underwent long luteal gonadotropin releasing hormone (GnRH) agonist protocol, were included. Patients with hydrosalpinx, organic pathology in the uterine cavity and anovulatory menstrual cycles were excluded. Since office hysteroscopy is a routine part of pre-procedural evaluation of the patients before the first IVF-ET cycle in the IVF clinic, in order to maximize the available number of patients that received LEI, these patients were selected as the study group.

Uterine cavities were evaluated by office hysteroscopy in the menstrual cycle's early proliferative phase before IVF-ET cycle. The study group involved 250 patients with no endometrial pathology detected during hysteroscopy and had LEI in three regions: anterior uterine wall, posterior wall and the fundus, using office hysteroscopic scissors. The control group consisted of 122 consecutive patients who only had a diagnostic office hysteroscopy with no endometrial pathology prior to this study and met the same inclusion criteria. Of- fice hysteroscopy and LEI procedures were carried out by an experienced reproductive endocrinologist to maintain uniform injury size and minimize potential bias.

For each patient, long luteal $\mathrm{GnRH}$ agonist protocol was used for controlled ovarian hyperstimulation $(\mathrm{COH})$. Combined oral contraceptive pill (Desolett; Schering Plow, USA) was administered on the third day of the last spontaneous menstrual cycle before the IVF-ET cycle. In order to suppress the pituitary gland and spontaneous ovulation, GnRH agonist (Lucrin; Abbott, France or Decapeptyl; Erkim Pharmaceuticals, Turkey) was administered on the $21^{\text {st }}$ day of the menstrual cycle. Once pituitary suppression was achieved, (serum Estradiol (E2) concentration $<40 \mathrm{pg} / \mathrm{ml}$ or no ovarian follicles $>10 \mathrm{~mm}$ ), the agonist dose was halved. Recombinant follicle stimulating hormone ( $\mathrm{rFSH}$ ) (Gonal-F; Serona, Italy or Puregon; Organon, Net herlands) or human menopausal gonadotropin (HMG) (Menogon; Ferring, Germany or Menopur; Ferring, Germany or Merional; ARISTA, Turkey) was administered for ovarian stimulation.

Follicular development was followed up via transvaginal ultrasonography (USG) $(8 \mathrm{MHz}$, Logiq, USA) and serum E2 measurements. Recombinant human chorionic gonadotropin (hCG) (Ovitrelle; Serona, Germany) 250 mcg or urinary hCG (Pregnyl, Netherlands) 10,000 IU was given when at least three follicles with a diameter $\geq 17 \mathrm{~mm}$ were detected. Oocyte pick-up (OPU) was performed under intravenous sedo-analgesia and transvaginal ultrasonographic guidance, approximately 36 hours after hCG administration. Follicular fluid and oocytes were aspirated with a pressure of $100-120 \mathrm{mmHg}$ (Lobetect, Germany) using 16-18-gauge needle, penetrating sharply into follicles larger than $10 \mathrm{~mm}$.

Embryo transfer was performed under transabdominal ultrasonographic (5 MHz, Logiq, USA) guidance 2-5 days after OPU, preferably 1-3 embryos of grade 1-2 quality, that were obtained using the standard procedure of intracytoplasmic sperm injection (ICSI) were transferred. Beginning with the OPU day, progesterone gel (8\% Crinone gel, Watson, USA) was used vaginally twice a day during the first 12 weeks of pregnancy, for luteal phase 
support. On the $12^{\text {th }}$ day after the embryo transfer, serum $\beta$-hCG was measured in order to evaluate treatment outcomes. Transvaginal USG was used on the $6^{\text {th }}$ week, for clinical pregnancy detection.

The primary goal of this study was to determine the impact of LEI on implantation rate; secondary goal was to determine its impact on clinical pregnancy and miscarriage rates. The number of gestational sacs observed by USG was divided to the total number of transferred embryos in order to calculate the implantation rate. Clinical pregnancy was identified as the presence of intrauterine gestational sac with a positive fetal heart activity on the $6^{\text {th }}$ week. In order to calculate the clinical pregnancy rate, the number of patients diagnosed with clinical pregnancy was divided by the total number of patients who underwent embryo transfer. Miscarriage rate was calculated by dividing the number of miscarriage before the $24^{\text {th }}$ week of gestation to the number of clinical pregnancies. ${ }^{3}$

SPSS for Windows 15.0 (SPSS for Windows, Chicago, Illinois) was used for statistical analysis. The student's t test was employed for the normally distributed quantitative data. Mann-Whitney U test was preferred for variables that did not fit the normal distribution; and, Chi-Square and FisherExact tests were utilized for qualitative and categorical data. Descriptive values include mean \pm

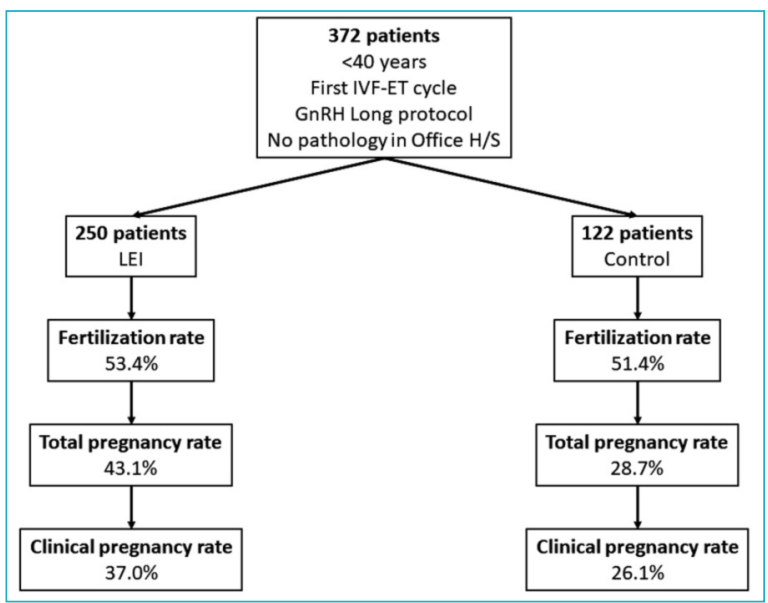

FIGURE 1: Flow-chart of the study.

standard deviation (SD), median and minimum / maximum values, frequency and percentage values for qualitative data. Statistical significance threshold was accepted as 0.05 .

\section{RESULTS}

Out of 372 patients evaluated, the study group consisted of 250 patients while the control group had 122 patients. Figure 1 demonstrates the flow-chart of the study. The main characteristics of the patients are shown in Table 1 . There was no statistically significant difference between the groups regarding the baseline characteristics. The patients'

\begin{tabular}{|c|c|c|c|}
\hline Variables & Local endometrial injury group $(n=250)$ & Control group $(n=122)$ & $\mathrm{p}$ \\
\hline Age, years $($ mean $\pm S D)$ & $28.0 \pm 4.3$ & $28.2 \pm 4.9$ & NS \\
\hline $\mathrm{BMI}, \mathrm{kg} / \mathrm{m}^{2}(\mathrm{mean} \pm \mathrm{SD})$ & $24.9 \pm 4.5$ & $25.1 \pm 4.2$ & NS \\
\hline Baseline FSH, IU/L (mean $\pm S D)$ & $6.5 \pm 2.3$ & $6.4 \pm 1.9$ & NS \\
\hline Baseline LH, IU/L (mean $\pm S D)$ & $6.2 \pm 3.7$ & $6.1 \pm 3.4$ & NS \\
\hline Baseline E2,pg/ml (mean \pm SD) & $43.7 \pm 20.1$ & $42.9 \pm 19.9$ & NS \\
\hline $\mathrm{AFC}\left(\mathrm{mean}_{ \pm} \mathrm{SD}\right)$ & $14.0 \pm 6.6$ & $13.4 \pm 6.6$ & NS \\
\hline \multicolumn{4}{|l|}{ Infertility etiologies, $n(\%)$} \\
\hline Male factor & $100(40.0 \%)$ & $56(45.9 \%)$ & \\
\hline Tubo-peritoneal factor & $10(4.0 \%)$ & $3(2.5 \%)$ & \\
\hline Endometriosis & $15(6.0 \%)$ & $3(2.5 \%)$ & NS \\
\hline Unexplained infertility & $58(23.2 \%)$ & $32(26.2 \%)$ & \\
\hline Decreased ovarian reserve & $67(26.8 \%)$ & $28(23.0 \%)$ & \\
\hline
\end{tabular}

AFC: Antral follicle counts; FSH: Follicle stimulating hormone; LH: Luteinizing hormone; SD: Standard deviation; E2: Estradiol; n: number; NS: Non-significant; BMI: Body mass index. 
mean age was $28 \pm 4.3$ years in the study group and $28.3 \pm 4.9$ years in the control group. In the LEI group, the indication for IVF-ET was male factor (100/250, 40.0\%), tubo-peritoneal factor (10/250, $4.0 \%)$, endometriosis $(15 / 250,6.0 \%)$, unexplained infertility $(58 / 250,23.2 \%)$ and decreased ovarian reserve $(67 / 250,26.8 \%)$. In the control group, distribution of the indications for IVF were similar to the study group; male factor (56/122, 45.9\%), tuboperitoneal factor $(3 / 122,2.5 \%)$, endometriosis $(3 / 122,2.5 \%)$, unexplained infertility $(32 / 122$, $26.2 \%)$, and decreased ovarian reserve (28/122, $23.0 \%)$.

Table 2 demonstrates the clinical features of the controlled ovarian hyperstimulation $(\mathrm{COH}) \mathrm{cy}-$ cles of the patients participating in the study. The mean duration of the stimulation was statistically significantly longer in the LEI group $(9.5 \pm 1.8$ days versus and $8.7 \pm 2.1$ days, $p<0.001$ ), while the mean total gonadotropin dose used per cycle was significantly lower $(1990 \pm 737$ IU versus $2530 \pm 1082$ IU, respectively, $\mathrm{p}<0.001)$ than the control group.
On the day of hCG, the mean endometrial thickness was $10.2 \pm 2.1 \mathrm{~mm}$ in the study group and $9.9 \pm 2.2 \mathrm{~mm}$ in the control group, and the groups did not reflect any statistically significant difference. The difference between the mean endometrial thickness of the two groups on the OPU day, was statistically significant, since it was $9.4 \pm 3.1 \mathrm{~mm}$ in the study group and $10.3 \pm 2.3 \mathrm{~mm}$ in the control group. In the study group, 181 patients underwent embryo transfer and this number was 92 in the control group. In the study group, the mean endometrial thickness on ET day was significantly lower than the control group $(9.6 \pm 4.0 \mathrm{~mm}$ versus11.1 $\pm 2.2 \mathrm{~mm}$, respectively, $\mathrm{p}<0.001)$.

The clinical features of oocytes and embryos are shown in Table 3. The difference was not statistically significant between the two groups with regard to total oocyte count, number of oocytes in metaphase II and number of oocytes undergoing ICSI. The number of embryos transferred was significantly higher in the control group $(\mathrm{p}<0.001)$. Fertilization rate was $53.4 \%$ in the study group and

TABLE 2: Clinical features of the controlled ovarian hyperstimulation cycles in the study and the control group.

\begin{tabular}{|lccc|}
\hline & $\begin{array}{c}\text { Local endometrial injury group } \\
\text { Variables }\end{array}$ & $\begin{array}{c}\text { Control group } \\
\text { mean } \pm \text { SD }\end{array}$ & $\mathbf{p}$ \\
Duration of stimulation, (days) & $9.5 \pm 1.8$ & $8.7 \pm 2.1$ & $<0.001$ \\
\hline Total dose of gonadotropin, $(\mathrm{IU})$ & $1990 \pm 737$ & $2530 \pm 1082$ & $<0.001$ \\
Endometrial thickness on the day of $\mathrm{hCG},(\mathrm{mm})$ & $10.2 \pm 2.1$ & $9.9 \pm 2.2$ & $\mathrm{NS}$ \\
\hline Endometrial thickness on OPU day, $(\mathrm{mm})$ & $9.4 \pm 3.1$ & $10.3 \pm 2.3$ & $<0.05$ \\
Endometrial thickness on ET day, $(\mathrm{mm})$ & $9.6 \pm 4.0$ & $11.1 \pm 2.2$ & $<0.001$ \\
\hline
\end{tabular}

hCG: Human chorionic gonadotropin; COH: Controlled ovarian hyperstimulation; OPU: Oocyte pick-up; SD: Standard deviation; ET: Embryo transfer; NS: Non-significant.

TABLE 3: Outcome of controlled ovarian hyperstimulation cycles in the study and the control group.

\begin{tabular}{|c|c|c|c|}
\hline & $\begin{array}{l}\text { Local endometrial injury group } \\
\text { median (min-max) }\end{array}$ & $\begin{array}{c}\text { Control group } \\
\text { median (min-max) }\end{array}$ & $p$ \\
\hline Total oocyte counts & $13(1-51)$ & $14(1-43)$ & NS \\
\hline Number of oocytes in metaphase II & $10(0-38)$ & $10(0-32)$ & NS \\
\hline Number of oocytes that underwent ICSI & $11(0-45)$ & $12(0-36)$ & NS \\
\hline Number of embryos transferred & $2(0-3)$ & $3(0-3)$ & $<0.001$ \\
\hline Fertilization rate & $53 \%$ & $51 \%$ & NS \\
\hline D3 ET count & $100(35.5 \%)$ & $59(55.2 \%)$ & $<0.01$ \\
\hline D5 ET count & $182(64.5 \%)$ & $48(44.8 \%)$ & 0.014 \\
\hline
\end{tabular}

ICSI: Intracytoplasmic sperm injection; ET: Embryo transfer; NS: Non-significant. 
TABLE 4: Outcome of controlled ovarian hyperstimulation cycles in the study and the control group.

\begin{tabular}{|lccc|}
\hline & Local endometrial injury group & Control group & p \\
\hline Implantation rate & $19 \%$ & $12.9 \%$ & NS \\
\hline Clinical pregnancy rate & $28.7 \%$ & $26.1 \%$ & NS \\
\hline Total pregnancy rate & $43.1 \%$ & $37 \%$ & NS \\
\hline Miscarriage rate & $12.5 \%$ & $9.6 \%$ & NS \\
\hline
\end{tabular}

NS: Non-significant.

$51.4 \%$ in the control group. The difference between the groups was not statistically significant in terms of fertilization rates. One hundred (35.5\%) D3 and $182(64.5 \%)$ D5 transfers were carried out in the study group, while these numbers were 59 $(55.2 \%)$ and $48(44.8 \%)$ in the control group.

In the study group, total and clinical pregnancy rates were higher ( $43.1 \%$ vs $37 \%, \mathrm{p}>0.05$, and $28.7 \%$ vs $26.1 \%$ p $>0.05$, respectively). However, there was no statistically significant difference between the groups. Also the difference between the groups was not statistically significant in terms of miscarriage rates. The implantation rates were similar (19\% and $12.9 \%$, respectively) as well (Table 4).

\section{DISCUSSION}

In this study, which included the patients who were under 40 years of age in their first IVF-ET cycle; LEI was carried out by office hysteroscopy through the early proliferative phase before the IVF-ET cycle. Than the impact of the LEI on pregnancy and implantation rates were evaluated. In the study group, there was an increase in the clinical pregnancy, total pregnancy and implantation rates, but there was no statistically significant difference between the groups. Since office hysteroscopy is a routine part of pre-procedural evaluation of the patients before the first IVF-ET cycle in our IVF clinic, in order to maximize the available number of patients that received LEI, these patients were selected as the study group.

The first studies investigating the effect of LEI on the success of IVF-ET cycle were conducted in patients who had recurrent IVF failure. The earliest of these was reported by Barash et al. in 2003. A total of 134 patients with recurrent IVF failure were evaluated in that study. ${ }^{3}$ Of them, 45 underwent endometrial sampling with biopsy catheter on days $8,12,21$ and 26 of the cycle prior to the IVF-ET cycle, and it was shown that clinical pregnancy, implantation, and live birth rates were doubled. After this study, in 2007, Raziel et al., performed LEI on 60 women who had recurrent IVF failure, on the $21^{\text {st }}$ and $26^{\text {th }}$ days of the luteal phase, and implantation and pregnancy rates were higher than the control group according to their findings. ${ }^{9}$ After these two non-randomized trials, randomized controlled trials evaluating the effect of endometrial injury in women who had recurrent IVF failure were published. In most of these studies, LEI in the cycle preceding the IVF-ET cycle increased significantly in terms of clinical pregnancy, implantation and/or live birth rates, while it was found ineffective in other studies. ${ }^{10-14}$

In two meta-analyses, in which randomized and non-randomized trials were evaluated together, it was stated that LEI on women who had recurrent IVF failure increased the clinical pregnancy rates with respect to the control group. ${ }^{4,5}$ In the review of 14 studies, LEI was found to be related with an increase in clinical pregnancy rates in women who had two or more ET, while having no effect on incidence of twin pregnancy, miscarriage or bleeding. ${ }^{15}$ In the current study, it was found that LEI increased implantation and clinical pregnancy rates in the patients who had their first IVF-ET cycle but this increase was not statistically significant.

The first study investigating the impact of LEI in the first IVF-ET cycle was reported by Karimzade et al. in 2010. In this prospective randomized controlled trial, 77 patients with the first IVF-ET cycle had two endometrial biopsies taken from the anterior and posterior walls of the uterus 
with Novak curette on the OPU day. ${ }^{6}$ Clinical pregnancy rates (12.3\% vs $32.9 \%$; odds ratio [OR] 0.25; 95\% confidence interval $[\mathrm{CI}]=0.12-0.66)$, implantation rates $(7.9 \%$ vs $22.9 \%)$ and ongoing pregnancy rates $(9.6 \%$ vs $29.1 \%$; OR $0.25 ; 95 \% \mathrm{CI}=$ $0.10-0.64$ ) were found to be significantly lower in the study group. ${ }^{6}$ However, the current study demonstrated that LEI conducted in early proliferative phase, performed prior to the IVF-ET cycle increased the implantation rates (19\% vs $12 \%$ ).

In a prospective randomized controlled study, 400 patients with the first IVF-ET cycle had LEI performed on them with a biopsy catheter in the luteal phase before the IVF-ET cycle. Live birth and implantation rates were significantly lower in the control group than the study group $(67 \%$ vs $28 \%$, and $22.4 \%$ vs $18 \%$, respectively). ${ }^{16}$ In 2014 , Yeung et al., evaluated 209 sub-fertile women with the first IVF-ET cycle, who underwent LEI with a biopsy catheter in the luteal phase preceding the IVF-ET cycle and no difference was reported in ongoing pregnancy rates of the study and control groups. ${ }^{17}$ In these two studies, unlike our study, LEI was induced by a biopsy catheter in the luteal phase of the menstrual cycle.

Although there is some evidence that carrying out LEI in the luteal phase leads to more decidualization and increases endometrial receptivity, it is still unclear whether it is better to perform on the luteal or proliferative phase. In this study, LEI was formed in the early proliferative phase similarly to the study of Huang et al. However, in some studies endometrial injury was created either both in the early proliferative phase with the luteal phase or only in the luteal phase. ${ }^{3,9-11,18}$

There is no consensus on the appropriate time interval between LEI and stimulation. According to our clinical protocol, injury was created in the menstrual cycle before stimulation, similar to some studies in the literature. ${ }^{3,9,10}$ However, there are some studies where LEI is performed during the COH cycle. ${ }^{6,18,19}$ In 2008, Zhou et al., reported increased live birth, implantation, and clinical pregnancy rates when they performed LEI in the stimulation cycle. This finding may be explained by the fact that LEI regresses the development of the endometrium that is under the effect of supraphysiological hormone levels and synchronizes the endometrium and embryo. ${ }^{19}$ However, Karimzade et al. showed that LEI induced by endometrial biopsy taken on the OPU day significantly reduced implantation and clinical pregnancy rates compared to the control group, which means that, LEI during stimulation cycle could adversely affect embryo implantation. They stated that LEI in the luteal phase before stimulation cycle induced gene expression, increased cytokine production and other positive effects, whereas the protein profile created by LEI during stimulation cycle might have a negative effect on embryo and receptive endometrium and therefore a certain interval between injury and transfer was required. ${ }^{6}$

The retrospective nature of this study is its major limitation. Another important limitation is the inadequate homogenization of the patients, especially in terms of embryo transfer days. Larger scale studies in which variables other than LEI can be controlled, would be more valuable.

\section{CONCLUSION}

In conclusion, although it was found that LEI slightly increased clinical pregnancy and implantation rates in women with first IVF-ET cycle, there was no statistically significant difference between the groups. The outcome of ongoing prospective randomized controlled trials that aim to investigate the clinical outcome of LEI prior to the IVF cycle may give more concrete data. ${ }^{20-22}$

\section{Source of Finance}

During this study, no financial or spiritual support was received neither from any pharmaceutical company that has a direct connection with the research subject, nor from a company that provides or produces medical instruments and materials which may negatively affect the evaluation process of this study.

\section{Conflict of Interest}

No conflicts of interest between the authors and / or family members of the scientific and medical committee members or members of the potential conflicts of interest, counseling, expertise, working conditions, share holding and similar situations in any firm. 


\section{Authorship Contributions}

Idea/Concept: Zeliha Firat Cuylan, Berfu Demir, Berna Dilbaz; Design: Serdar Dilbaz, Berfu Demir, Berna Dilbaz; Control/Supervision: Serdar Dilbaz, Berfu Demir, Berna Dilbaz; Data Collection and/or Processing: Zeliha Firat Cuylan, Berfu Demir;
Analysis and/or Interpretation: Zeliha Firat Cuylan, Berfu Demir, Berna Dilbaz; Literature Review: Zeliha Firat Cuylan, Berfu Demir; Writing the Article: Zeliha Furat Cuylan, Berfu Demir, Berna Dilbaz, Serdar Dilbaz; Critical Review: Berna Dilbaz, Serdar Dilbaz; Materials: Zeliha Firat Cuylan, Berfu Demir.

\section{REFERENCES}

1. Nyboe Andersen A, Goossens V, Bhattacharya S, Ferraretti AP, Kupka MS, de Mouzon J, et al. Assisted reproductive technology and intrauterine inseminations in Europe, 2005: results generated from European registers by ESHRE: ESHRE. The European IVF Monitoring Programme (EIM), for the European Society of Human Reproduction and Embryology (ESHRE). Hum Reprod. 2009;24(6):1267-87. [Crossref] [PubMed]

2. Vélez MP, Connolly MP, Kadoch IJ, Phillips S, Bissonnette $F$. Universal coverage of IVF pays off. Hum Reprod. 2014;29(6):1313-9. [Crossref] [PubMed]

3. Barash A, Dekel N, Fieldust S, Segal I, Schechtman E, Granot I. Local injury to the endometrium doubles the incidence of successful pregnancies in patients undergoing in vitro fertilization. Fertil Steril. 2003;79(6):131722. [Crossref] [PubMed]

4. El-Toukhy T, Sunkara S, Khalaf Y. Local endometrial injury and IVF outcome: a systematic review and meta-analysis. Reprod Biomed Online. 2012;25(4):345-54. [Crossref] [PubMed]

5. Potdar N, Gelbaya T, Nardo LG. Endometrial injury to overcome recurrent embryo implantation failure: a systematic review and metaanalysis. Reprod Biomed Online. 2012;25(6):561-71. [Crossref] [PubMed]

6. Karimzade MA, Oskouian $\mathrm{H}$, Ahmadi S, Oskouian L. Local injury to the endometrium on the day of oocyte retrieval has a negative impact on implantation in assisted reproductive cycles: a randomized controlled trial. Arch Gynecol Obstet. 2010;281(3):499-503. [Crossref] [PubMed]

7. Simón C, Bellver J. Scratching beneath 'The Scratching Case': systematic reviews and meta-analyses, the back door for evidencebased medicine. Hum Reprod. 2014;29(8):1618-21. [Crossref] [PubMed]

8. Santamaria X, Katzorke N, Simón C. Endometrial 'scratching': what the data show. Curr Opin Obstet Gynecol. 2016;28(4):242-9.
[Crossref] [PubMed]

9. Raziel A, Schachter M, Strassburger D, Bern $O$, Ron-El R, Friedler S. Favorable influence of local injury to the endometrium in intracytoplasmic sperm injection patients with highorder implantation failure. Fertil Steril. 2007;87(1):198-201. [Crossref] [PubMed]

10. Karimzadeh MA, Ayazi Rozbahani M, Tabibnejad N. Endometrial local injury improves the pregnancy rate among recurrent implantation failure patients undergoing in vitro fertilisation/intra cytoplasmic sperm injection: a randomised clinical trial. Aust N Z J Obstet Gynaecol. 2009;49(6):677-80. [Crossref] [PubMed]

11. Narvekar SA, Gupta N, Shetty N, Kottur A, Srinivas M, Rao KA. Does local endometrial injury in the nontransfer cycle improve the IVFET outcome in the subsequent cycle in patients with previous unsuccessful IVF? A randomized controlled pilot study. J Hum Reprod Sci. 2010;3(1):15-9. [Crossref] [PubMed] [PMC]

12. Gibreel A, Badawy A, El-Refai W, El-Adawi N Endometrial scratching to improve pregnancy rate in couples with unexplained subfertility: a randomized controlled trial. J Obstet Gynaecol Res. 2013;39(3):680-4. [Crossref] [PubMed]

13. Baum M, Yerushalmi GM, Maman E, Kedem A, Machtinger R, Hourvitz A, et al. Does local injury to the endometrium before IVF cycle really affect treatment outcome? Results of a randomized placebo controlled trial. Gynecol Endocrinol. 2012;28(12):933-6. [Crossref] [PubMed]

14. Tk $A$, Singhal $H, S$ Premkumar $P$, Acharya $M$, $S$ Kamath K, George K. Local endometrial injury in women with failed IVF undergoing a repeat cycle: a randomized controlled trial. Eur J Obstet Gynecol Reprod Biol. 2017;214:10914. [Crossref] [PubMed]

15. Nastri CO, Lensen SF, Gibreel A, Raine-Fenning N, Ferriani RA, Bhattacharya S, et al. Endometrial injury in women undergoing assisted reproductive techniques. Cochrane Database
Syst Rev. 2015(3):CD009517. [Crossref] [PubMed]

16. Mahran A, Ibrahim M, Bahaa $\mathrm{H}$. The effect of endometrial injury on first cycle IVF/ICSI outcome: a randomized controlled trial. Int $\mathrm{J}$ Reprod Biomed (Yazd). 2016;14(3):193-8. [Crossref] [PubMed]

17. Yeung TW, Chai J, Li RH, Lee VC, Ho PC, Ng $\mathrm{EH}$. The effect of endometrial injury on ongoing pregnancy rate in unselected subfertile women undergoing in vitro fertilization: a randomized controlled trial. Hum Reprod. 2014;29(11):2474-81. [Crossref] [PubMed]

18. Huang SY, Wang CJ, Soong YK, Wang HS, Wang ML, Lin CY, et al. Site-specific endometrial injury improves implantation and pregnancy in patients with repeated implantation failures. Reprod Biol Endocrinol. 2011;9:140. [Crossref] [PubMed] [PMC]

19. Zhou L, Li R, Wang R, Huang HX, Zhong $K$. Local injury to the endometrium in controlled ovarian hyperstimulation cycles improves implantation rates. Fertil Steril. 2008;89(5):116676. [Crossref] [PubMed]

20. Lensen S, Martins W, Nastri C, Sadler L, Farquhar C. Pipelle for Pregnancy (PIP): study protocols for three randomised controlled trials. Trials. 2016;17(1):216. [Crossref] [PubMed] [PMC]

21. Pye C, Chatters R, Cohen J, Brian K, Cheong YC, Laird S, et al. Induced endometrial trauma (endometrial scratch) in the mid-luteal menstrual cycle phase preceding first cycle IVF/ICSI versus usual IVF/ICSI therapy: study protocol for a randomised controlled trial. BMJ Open. 2018;8(5):e020755. [Crossref] [PubMed] [PMC]

22. van Hoogenhuijze NE, Torrance HL, Mol F, Laven JSE, Scheenjes E, Traas MAF, et al. Endometrial scratching in women with implantation failure after a first IVF/ICSI cycle; does it lead to a higher live birth rate? The SCRaTCH study: a randomized controlled trial (NTR 5342). BMC Womens Health. 2017;17(1):47. [Crossref] [PubMed] [PMC] 